\title{
Isthmocele - To Treat or Not to Treat?
}

\author{
Baranowski W, Doniec J and Szafarowska M* \\ Department of Gynecology and Oncological Gynecology, Military Institute of Medicine, Poland
}

*Corresponding author: Monika Szafarowska, Department of Gynecology and Oncological Gynecology, Military Institute of Medicine, 04-141 Warsaw, 128 Szaserów Street, Poland.

To Cite This Article: Szafarowska M, Isthmocele - To Treat or Not to Treat?. 2020 - 7(6). AJBSR.MS.ID.001202.

DOI: 10.34297/AJBSR.2020.07.001202.

Received: 眥 February 24, 2020; Published: 制 March 03, 2020

\begin{abstract}
Due to the growing number of Cesarean section deliveries, the uterine scar defect is becoming a common problem in gynecology and in obstetrical care. The symptoms of the niche include abnormal uterine bleeding (AUB, 65\%), spotting between menses (76\%), dysmenorrhea (53.1\%), chronic pelvic pain (46.2\%), infertility (71.5\%) and dyspareunia (24.2\%). The diagnosis of isthmocele is based on the imaging methods performed during the early proliferative phase of the menstrual cycle: transvaginal ultrasound (TVUS), saline infusion sonography (SIS), magnetic resonance imaging or hysteroscopy. The treatment of niche includes conservative procedures like management with expectant, oral contraceptive or hormonal IUD (levonorgestrel-releasing device). In case of incidental diagnosis of isthmocele (asymptomatic) there is no indication for any treatment. Surgical treatments include endoscopic (hysteroscopy, laparoscopy) procedures, repair by laparotomy, transvaginal or combined abdominal/transvaginal operations. The decision to treat and how to treat is made individually, considering the severity of the condition and the patients' desire for future fertility. Hysteroscopic treatment should be considered as a first line surgical treatment in fertility desire women, because of minimally invasive and beneficial therapeutic results. It is associated with nearly $100 \%$ of efficacy in reducing the AUB symptoms and in restoring fertility. The laparoscopic, laparotomic or vaginal repair techniques are dedicated to fertility desire women with residual myometrial thickness $<5 \mathrm{~mm}$. According to literature data about $90 \%$ of the patients had resolution of their symptoms and the percentage of patients with restoring fertility after treatment was about $44 \%$. However, there is no data available to confirm the effectiveness of treatment in preventing the obstetrical complications like uterine rupture or pregnancy in the scar.
\end{abstract}

Keywords: Isthmocele; Niche; Residual myometrial thickness; Transvaginal ultrasound; Infertility; UB; Hysteroscopy

Abbreviations: RMT: Residual Myometrial Thickness; TVUS: Transvaginal Ultrasound; SHG: Sonohysterography; AUB: Abnormal Uterine Bleeding; IUD: Intrauterine Device

\section{Introduction}

Due to the growing number of Cesarean section deliveries, the uterine scar defect is becoming an increasingly problem in gynecology and in obstetrical care. According to epidemiological data based on transvaginal ultrasound (TVUS) or sonohysterography (SHG) examination, the Cesarean scar defects are very frequent between $24 \%$ and $84 \%$ of women will develop it [1]. Cesarean scar defect is described in literature as isthmocele or niche but also as diverticulum, pouch, sacculatio, dehiscentio or hernia.

\section{The Symptoms and The Goals of Treatment}

The first description of the isthmocele dates back to 1975, but precise and detailed description of abnormalities was published in 1995 by H. Morris who found the thinning and widening of the scar and forms a pocket, lymphocytic inflammation, containing residual suturing material, abnormal endometrium and dilated vessels. From clinical point of view, isthmocele at least $2 \mathrm{~mm}$ deep and $4 \mathrm{~mm}$ wide seems to be relevant and symptomatic [2]. In papers published so far, many factors associated with the risk of isthmocele were analyzed, but without conclusion. The most important risk factor appears to be multiple previous Cesarean sections, furthermore the incompetent closure of the uterine incision related to suturing technique: single layer, non-perpendicular sutures or endometrial/decidua omitting techniques may also be a cause of the defect [1]. The retroverted uterus seems to be also a risk factor of isthmocele - due to greater mechanical traction which leads to blood perfusion disorders, than compromised wound healing. Furthermore, individual predispositions or habits like diabetes, 
cigarettes smoking or early post-operative uterine adhesions with anterior abdominal wall can also cause isthmocele [3].

The most common symptoms of the niche include abnormal uterine bleeding (AUB, 65\%), spotting between menses (76\%), dysmenorrhea (53.1\%), chronic pelvic pain (46.2\%), infertility $(71.5 \%)$ and dyspareunia $(24.2 \%)(1,3)$. These symptoms are probably due to intermittent passage of retained menstrual blood within the isthmocele. In addition, chronic inflammation and the presence of endometrium within niche also can induce abnormal bleeding. Probably in women presenting niche, infertility is a consequence of impaired endometrial receptivity, induced by abnormal pH (more acidic) in the uterine cavity and inflammation in niche. Furthermore, the isthmocele adversely affects subsequent pregnancy and is associated with complications including: cesarean scar pregnancy (1/188 6-2216), uterine rupture risk - 2\% when residual myometrial thickness (RMT), is $>50 \%$ of total uterine width and $5 \%$ when RMT is $<50 \%$, placenta increta or placenta previa $[1,3]$.

The diagnosis of isthmocele is based on the imaging methods performed during the early proliferative phase of the menstrual cycle: transvaginal ultrasound (TVUS), saline infusion sonography
(SIS) or magnetic resonance imaging, sometimes also diagnostic hysteroscopy. Isthmocele is described as a triangular, hypoechoic defect at the site of hysterotomy on the anterior uterine wall. According to existing data SIS is the most accurate (most sensitive and specific) method to identify and measure a niche, SIS also facilitate better measurement of the residual myometrium thickness (RMT), what is the most important clinical factor imposing further decisions [1].

The treatment of niche includes conservative procedures like management with expectant, oral contraceptive or hormonal IUD (levonorgestrel-releasing device). In case of incidental diagnosis of isthmocele (asymptomatic) there is no indication for any treatment. Surgical treatments include endoscopic (hysteroscopy, laparoscopy) procedures, repair by laparotomy, transvaginal or combined abdominal/transvaginal operations $[1,4,5]$. The decision to treat and how to treat is made individually, considering the severity of the condition and the patients' desire for future fertility. The main goal of the clinical management of niche is to reduce the symptoms of AUB, to restore fertility and to prevent uterine scar rupture during pregnancy. A diagram of the isthmocele treatment for symptomatic women is presented in Figure 1.

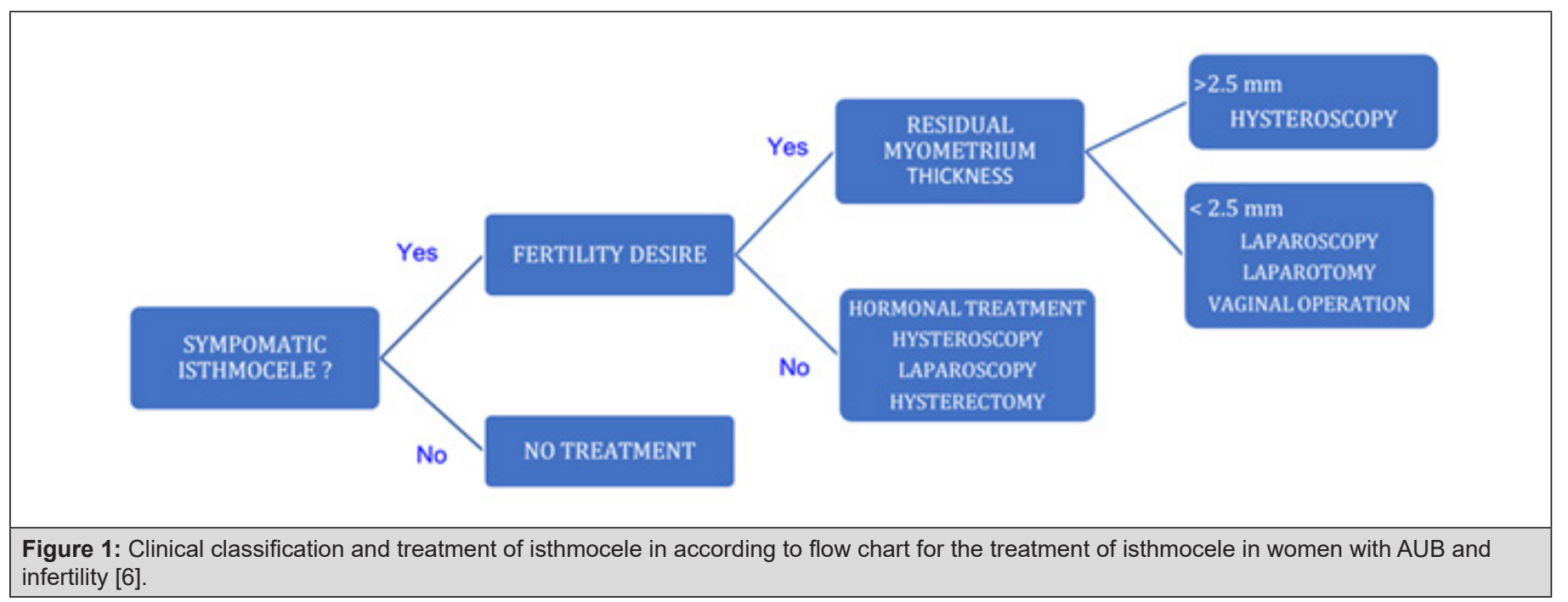

Comparing the results of the conservative treatment with OC or hormonal IUD vs hysteroscopy, literature data showed a significantly greater efficacy of hysteroscopy in reducing the symptoms of AUB [5-7]. Hysteroscopic treatment should be considered as a first line surgical treatment because of minimally invasive and beneficial therapeutic results. The most used technique for the hysteroscopic treatment of isthmocele is the resection of the distal edge of the scar defect, and the destruction (coagulation) of abnormal scar tissue containing endometrium and dilated vessels. This treatment may be considered in symptomatic women when RMT $>50 \%$ or $>3 \mathrm{~mm}$. The flattening of the distal edge of the scar enables to reduce the amount of residual menstrual blood. The use of a hysteroscope with a $9 \mathrm{~mm}$ diameter, together with additional devices such as rollerball allowing ablation the abnormal tissue, is associated with nearly $100 \%$ of efficacy in reducing the AUB symptoms and in restoring fertility [5,7]. More advanced cases of niche with RMT < $50 \%$ or $<3 \mathrm{~mm}$, usually require surgical resection through vaginal, laparoscopic, laparotomic or combined (abdominal/transvaginal) approaches.

The vaginal approach to isthmocele repair is technically difficult due to the limited field of the operation. This technique requires the bladder preparation, opening the vesicovaginal space and resection of fibrotic cesarean scar. Intraoperative identification of the defect is the most difficult part of the operation. Additional 
devices like hysteroscope (backlight), Foley catheter, or Hegar dilators ("slip and hook" technique) are very useful for the mapping the defect during these procedures. The gentle tissue preparation and non-coagulating cutting, followed by two-layer closures (2-0 thread) appeared to be the most effective way of treatment. The improvement of reducing the symptoms was reported in nearly $90 \%[5,8]$.

Laparoscopic approach for surgical treatment of isthmocele for the first time was described by O. Donnez in 2008. As in the vaginal approach, it is crucial to identify the defect on the anterior uterine wall. Surgical excision could be performed using a laser, monopolar electrode devices or scissors and double-layer closure is recommended. According to literature data about $90 \%$ of the patients had resolution of their symptoms and the percentage of patients with restoring fertility after treatment was about $44 \%$ $[4,5,9]$.

Abdominal approach (laparotomy, mini laparotomy) allows the operation to be carried out similarly to a laparoscopic surgery. As above mentioned, the edge to edge double-layer closure is highlighted. Some authors suggest that the technique allowing antepositioning of retroverted uterus (e.g. shortening of the round ligaments) is associated with better results. The pregnancies obtained after treatment with laparotomy are about 71\% [4]. Unfortunately, there is no data available to confirm the effectiveness of treatment in preventing the obstetrical complications like uterine rupture or pregnancy in the scar. Most of the treatment procedures described in the literature, concerns the women with infertility and women with abnormal uterine bleeding [10].

According to our experiences dealing with niche repair, in selected patients we recommend using office hysteroscopy devices (4-5 $\mathrm{mm}$ in diameter) as described previously by S. Bettocchi This procedure is restricted to the patients qualified to assisted reproduction techniques with niche in non-retroverted uterus, few days before insemination or embryo transfer. The main goal of this procedure is to reduce the niche debris by washing or gentle suction (usually 2 times in 2 days intervals), aiming that isthmocele acts as a "third sactosalpinx", thus disturbing infertility treatment.

\section{Conclusion}

Growing number of cesarean sections is strictly connected with specific pathological changes usually named isthmocele or niche. The concise epidemiological data, clinical presentation and possible risk factors are described. Controversy concerned the different methods of symptomatic niche treatment are discussed and authors experience in modified methods of hysteroscopic surgery of isthmocele is presented.

\section{Conflict of Interest}

No conflict of interest is declared by authors.

\section{References}

1. Bij de Vaate AJ, Van der Voet LF, Naji O, Witmer M, Veersema S, et al. (2014) Prevalence, potential risk factors for development and symptoms related to the presence of uterine niches following Cesarean section: systematic review. Ultrasound Obstet Gynecol 43(3): 372-382.

2. Morris H (1995) Surgical pathology of the lower uterine segment caesarean section scar: is the scar a source of clinical symptoms? Int J Gynecol Pathol 14(1): 16-20.

3. Kermer T, Ghiorzi IB, Dibi RP (2019) Isthmocele: an overwiev of diagnosis and treatment. Rev Assoc Med Bras 65(5): 714-721.

4. Setubal A, Alves J, Osório F, Guerra A, Fernandes R, et al. (2018) Treatment for uterine isthmocele, a pouchlike defect at the site of a cesarean section scar. J Minim Invasive Gynecol 25(1): 38-46.

5. Zhang X, Yang M, Wang Q, Chen J, Ding J, et al. (2016) Prospective evaluation of five methods used to treat cesarean scar defects. Int J Gynaecol Obstet 134(3): 336-339.

6. Vitale S, Ludwin A, Vilos GA, Török P, Tesarik J, et al. (2020) From hysteroscopy to laparoendoscopic surgery: what is the best surgical approach for symptomatic isthmocele? A systematic review and metaanalysis. Arch Gynecol Obstet 301(1): 33-52.

7. Florio P, Gubbini G, Marra E, Dores D, Nascetti D, et al. (2011) A retrospective case-control study comparing hysteroscopic resection versus hormonal modulation in treating menstrual disorders due to isthmocele. Gynecol Endocrinol 27(6): 434-438.

8. Chen Y, Chang Y, Yao S (2014) Transvaginal management of cesarean section diverticulum: a novel surgical treatment. Med Sci Monitor 20: 1395-1399.

9. Donnez O. Donnez J, Orellana R, Dolmans MM (2017) Gyneological and obstetrical outcomes after laparoscopic repair of cesarean scar defect of series of 38 women. Fertil Steril 107(1): 289-296.

10. Stegwee S, Jordans IPM, van der Voet LF, Bongers MY, De Groot CJM, et al. (2019) Single - versus double layer closure of the caesarean (uterine) scar in the prevention of gynaecological symptoms in relations to niche development - 2Close study: a multicentre randomised controlled trial. BMC Pregnancy Childbirth 19(1): 85. 